\title{
BRAIN NATRIURETIC PEPTIDE (BNP): BIOMARKER FOR RISK STRATIFICATION AND FUNCTIONAL RECOVERY PREDICTION IN ISCHEMIC STROKE
}

\author{
Ioana Stanescu ${ }^{1 / 2}$, Gabriela Dogaru ${ }^{1 / 2}$ \\ I" Iuliu Hațieganu" University of Medecine and Pharmacy Cluj-Napoca \\ ${ }^{2}$ Clinical Rehabilitation Hospital Cluj- Napoca
}

\begin{abstract}
Functional outcome after cardiovascular and cerebrovascular events is traditionally predicted using demographic and clinical variables like age, gender, blood pressure, cholesterol levels, diabetes status, smoking habits or pre-existing morbidity. Identification of new variables will improve the risk stratification of specific categories of patients. Numerous blood-based biomarkers associated with increased cardiovascular risk have been identified; some of them even predict cardiovascular events. Investigators have tried to produce prediction models by incorporating traditional risk factors and biomarkers. (1). Widely-available, rapidly processed and less expensive biomarkers could be used in the future to guide management of complex cerebrovascular patients in order to maximize their recovery (2)

Recently, studies have demonstrated that biomarkers can predict not only the risk for a specific clinical event, but also the risk of death of vascular cause and the functional outcome after cardiovascular or cerebrovascular events.

Early prediction of fatal outcome after stroke may improve therapeutic strategies (such as the use of more aggressive treatments or inclusion of patients in clinical trials) and guide decision-making processes in order to maximize patient's chances for survival and recovery. (3)

Long term functional outcome after stroke is one of the most difficult variables to predict. Elevated serum levels of brain natriuretic peptide (BNP) are powerful predictor of outcomes in patients with cardiovascular disease (heart failure, atrial fibrillation). Potential role of BNP in predicting atrial fibrillation occurrence, cardio-embolic stroke and post-stroke mortality have been proved in many studies. However, data concerning the potential role of BNP in predicting short term and long term functional outcomes after stroke remain controversial.
\end{abstract}

Keywords: brain natriuretic peptide, stroke, functional outcome, mortality prediction

Functional recovery after stroke is one of the most difficult variables to predict. Traditional models of outcome prediction included demographic and clinical parameters (such as age, gender, blood pressure, cholesterol levels, diabetes status, smoking habits or pre-existing conditions) or imagistic variables (such as size of infarction). Adding new variables to these models will improve the risk stratification of specific categories of patients. Numerous blood-based biomarkers associated with increased cardiovascular risk have been identified.
Many studies have demonstrate that biomarkers can predict not only the risk for occurrence of a specific event, but also the risk of death of vascular cause and the short term or long term functional outcome after cardiovascular or cerebrovascular events.

Multiple serum biomarkers were studied in correlation with cerebrovascular diseases: natriuretic peptides (mid-regional proatrial natriuretic peptide, brain natriuretic peptide $\mathrm{BNP}, \mathrm{N}$ terminalproBNP), sensitive cardiac Troponin, 
cystatin $\mathrm{C}$, cortisol, copeptin, interleukin6, C-reactive protein, D-dimers, etc).

Natriuretic peptides (NP) biological effects. NP are one of the most studied biomarkers in cardio-vascular diseases. Together with guanylin peptides and endogenous cardiac steroids, the natriuretic peptides belong to the group of natriuretic hormones, compounds that act in an endocrine or paracrine fashion to regulate extracellular fluid volume and blood pressure (BP) through the stimulation of sodium excretion by the kidney (4).

In addition, NP act as neurotransmitters or neuromodulators in the brain. B-type NP (BNP) was found in the hypothalamus and cerebral cortex, and was implicated in neuroprotection following brain injury. Elevated plasma BNP levels were described in patients with traumatic brain injury and stroke (4)

B-type natriuretic peptide (BNP) is a cardiac neurohormone, synthesized and released from the ventricular myocardium in response to increased wall tension such as volume or pressure overload. BNP has different systemic effects: vasodilatation, augmentation of urinary volume and sodium output, inhibition of sympathetic nervous system and of renin-angiotensinaldosterone system. (5).

BNP synthesis: B-type natriuretic peptide is synthesized as an inactive prohormone. It is cleaved into the bioactive hormone, BNP, which has an important role in cardiovascular remodelling and volume homeostasis, and the inactive $\mathrm{N}$-terminal fragment. Usually $\mathrm{BNP}$ is measured by this N-terminal fragment (NT-proBNP), since the Nterminal part seems to be more stable than BNP itself. Serum concentrations of NTproBNP are usually almost 10 fold higher than BNP concentrations; this is caused by differences in metabolism: the inactive fragment having a longer half-live (3).

\section{Serum BNP and NT-proBNP}

levels are variable, depending on physiological changes in cardiac, pulmonary and renal function; ageing, renal dysfunction and female gender were found to increase levels of natriuretic peptides

BNP levels are increased in a variety of non-cardiac conditions, because pro-inflammatory cytokines and neurohormones may stimulate its release: shock or other severe systemic conditions or even medication use could also influence BNP values (2).

NP levels in cardiological diseases: Increase in plasma levels of BNP and NT-proBNP are mainly related to cardiac pathology with impairment in left ventricular (LV) systolic or diastolic function. Thus, BNP measurement became a sensitive method to monitor LV function. BNP levels will increase in pathological cardiac conditions, such as left ventricular hypertrophy, ventricular dilatation, heart failure, acute coronary syndromes and atrial fibrillation (AF) (5).

The role of natriuretic peptides as powerful prognostic markers for cardiovascular outcomes and mortality was initially established in heart failure cohorts, thereafter in patients with acute coronary syndromes and later in stable coronary artery populations and in asymptomatic community-based elderly subjects. BNP is typically elevated in patients with heart failure (6).

Neuropeptides and risk factors. Higher levels of BNP and NT-proBNP are proven risk predictors in patients with and without cardio-vascular disease (1). Elevated plasma levels of NT-proBNB and of troponin $\mathrm{T}$ are markers of future major cardiac events, but also are correlated with multiple other vascular conditions and risk factors, even with mortality from noncardiovascular causes (7)

In neurovascular conditions, elevated plasma levels of BNP and NTproBNP predicts the risk for an incident ischemic stroke, predicts the risk for cardio-embolic etiology of a stroke, the risk of having a new onset AF episode in the setting of an ischemic stroke, the risk 
of death in the acute phase and the risk of poor functional recovery at 3 and 6 months after stroke. Generally, both BNP peptides have been associated with unfavorable clinical outcome and mortality in stroke patients (3)

In a prospective epidemiological study (7) was shown that increased levels of natriuretic peptides were associated positively with stroke risk factors and with increase in total stroke incidence : the combination of both elevated troponin $\mathrm{T}$ and NT-proBNP tripled the risk of ischemic stroke. BNP predicts a high-risk vascular profile in general population, risk factors for stroke were prevalent in individuals with high BNP values (7).

Although elevated troponin $\mathrm{T}$ and NT-proBNP should not be considered risk factors for ischemic stroke, their inclusion in " ecuations" used to predict stroke occurrence in general population might be useful. The addition of NT-proBNP to the $\mathrm{CHADS}_{2}$ and $\mathrm{CHA}_{2} \mathrm{DS}_{2}$-VASc risk stratification models resulted in significant improvements in the discrimination performance for both outcomes as well (8)

\section{BNP - biomarker for}

cardioembolic stroke: $\mathrm{AF}$ is a strong risk factor for ischemic -cardioembolic stroke. High levels of NT-proBNP are a strong predictor of atrial fibrillation, independent of other risk factors including echocardiographic parameters (8). There are arguments for NT-proBNP being of atrial origin in AF due to myocyte stress in the atria and thus reflecting atrial dysfunction (9). These facts lead to the conclusion that elevated levels of NTproBNP are markers for the cardioembolic stroke etiology. More than that, BNP levels strongly differentiate cardioembolic stroke subtype from all non-cardioembolic subtypes.

BNP and troponin $\mathrm{T}$ were associated with total stroke, nonlacunar ischemic, and especially, cardioembolic stroke occurrence in general population. ARIC study (7) demonstrate that $58 \%$ of cardioembolic strokes occurred in the highest quintile of NT-proBNP levels. We can extrapolate that high BNP values predicts the occurrence of a cardioembolic stroke type.

Among stroke subtypes, elevated BNP levels were found in $75 \%$ of cardioembolic strokes, $45,8 \%$ of lacunar strokes, $43,1 \%$ of large artery aterothrombotic strokes, and in $34,5 \%$ of cryptogenic strokes (10)

B-type natriuretic peptide levels have also been found to predict likelihood of subsequent identification of atrial fibrillation for patients with cryptogenic stroke. In cryptogenic stroke, high BNP level is an argument for cardioembolic etiology, and could influence the choice of an antithrombotic treatment. Elevated BNP levels can predict new AF in patients with acute ischemic stroke (11)

prediction.

BNP and stroke mortality

The plasma BNP level on admission can predict in-hospital death in patients with acute ischemic stroke (11): BNP level of $>240 \mathrm{pg} / \mathrm{ml}$ and NIHSS score $>13$ are associated with in-hospital mortality. Other authors used same combination of plasma NT-proBNP and NIHSS score in assessing in-hospital deaths: patients with NT-proBNP levels > $1583 \mathrm{pg} / \mathrm{ml}$ and NIHSS $>12,5$ on admission had a higher mortality rate (12)

In another study (13), BNP plasma levels were higher among patients who deteriorated , BNP level being an independent predictor of neurological worsening and death in the acute phase after stroke. BNP remained an independent predictor for death within 90 days, together with age and lesion size, in a study conducted by Nigro (14).

Levels of BNP and NT-proBNP measured in the acute stroke have been associated with mortality in a study conducted by Jensen: patients with NTproBNP levels $\leq 147 \mathrm{pg} / \mathrm{ml}$ had a better survival rate at 6 months (15). Levels of NT-proBNP were an independent predictor of 6 months mortality. 
In ARIC study, serum BNP levels independently predicted long term mortality and poorer functional outcome . BNP testing could identify those patients likely to require more aggressive treatment or intensive rehabilitation, having an important role in risk stratification of patients during admission and in acute phase. BNP could be also used to support early implementation of antithrombotic therapy, to determine the intensity of heart failure management in stroke patients, in order to improve their cardiac condition and, as a result, their rehabilitation potential (7).

BNP as a predictor of functional recovery after stroke. Functional prognosis after stroke is assessed using mainly the modified Rankin Scale (mRS). Good functional outcome was defined as mRS score 2 or less.

For short term prognosis after stroke, elevated BNP level is an independent marker for poor outcome at 90 days (10). Some authors postulate that the biomarker panel (BNP, NT-proBNP, cortisol and copeptin) predicted functional outcome and death within 90 days more efficiently than the NIHSS score (16)

BNP levels were found to be significantly higher in patients with larger infarcts and higher mRS scores (17). Higher BNP levels were independently associated with more proximal carotid axis occlusion, thus with larger infarct area (18)

BNP levels in patients with an unfavorable outcome after 90 days and after 1 year were significantly higher than those in patients with a favorable outcome (14). Same authors state that after adjustment in multivariate analyses, BNP did not remain an independent prognostic marker for functional outcome.

Elevated BNP levels on hospital admission for ischemic stroke will predict independently functional outcome at 6 months in a large, prospective cohort of patients (2) . BNP levels may have limited utility in assessing outcomes following non-cardioembolic stroke subtypes.
IN a study by Kerola (19) BNP was found to be a predictor for cognitive decline in an elderly general population.

In conclusion, in general population, elevated plasma levels of natriuretic peptides help to identify vascular high-risk individuals. In patients with ischemic stroke, increased BNP levels may confirm a cardioembolic etiology of the stroke. BNP and NTpro-BNP testing has a role in risk stratification of patients with ischemic stroke and may signal an unfavorable outcome, including the risk of death, at admission, on short term or long term, identifying those patients likely to require more aggressive treatment and more intensive early rehabilitation. 


\section{BIBLIOGRAPHY:}

1. Brown TM, Bittner v. Biomarkers of atherosclerosis: clinical applications. Curr Cardiol Rep Nov 2008; 10(6): 497-504

2. Rost NS, Biffi A, Cloonan L, Chorba J, Kelly P, Greer D, Ellinor P, Fune KL. Brain natriuretic peptide predicts functional outcome in ischemic stroke. Stroke. Feb 2012;43(2):441-445.

3. Garcia-Berrocoso T, Giralt D, Bustamante A, Etgen T, Jensen JK, Shamia J, Shibazaki K, Saritas A, Chen X, Whiteley WN, Montaner J. B-type natriuretic peptide and mortality afer stroke. Neurology. Dec 3. 2013; 81(23): 19761985

4. Hodes A, Lichtstein D. Natriuretic hormones and the brain. Front Endocrinol (Lausanne);2014;5:201

5. Femminella GD, deLucia $C$, Iacotucci $P$, Formisano R, Petraglia L, Alloca E,et al. Neuro-hormonal effects of physical activity in the eldery. Front Physiol.2013;4:378

6. Mukoyama M, Nakao K, Saito Y, Ogawa Y, Hosoda K, Suga S, Shirakami G, Jougasaki M, Imura $\mathrm{H}$. Increased human brain natriuretic peptide in congestive heart failure. $\mathrm{N}$ Eng1 $\mathrm{J}$ Med. 1990 Sep 13;323(11):757-8.

7. Folsom AR, Nambi V, Bell EJ, Oluleye OW, Gottesman RF, Lutsey P et al. Troponin $\mathrm{T}$, Nt-proBNP, and incidence of stroke: the atherosclerosis risk in communities (ARIC) study. Stroke. Apr. 2013; 44(4): 961-967

8. Patton KK, Ellinor PT, Heckbert SR, Christenson RH, DeFilippi C, Gottdiener JS, Kronmal RA. N-terminal pro-B-type natriuretic peptide is a major predictor of the development of atrial fibrillation: the Cardiovascular Health Study. Circulation 2009;120:1768-1774

9. Goetze JP, Friis-Hansen L, Rehfeld JF, Nilsson B, Svendsen JH. Atrial secretion of Btype natriuretic peptide. Eur Heart $J$ 2006;27:1648-1650

10. Chaudhuri JR, Sharma VK, Mridula KR, Balaraju B, Bandaru VC Association of plasma brain natriuretic peptide levels in acute ischemic stroke subtypes and outcome. J Stroke Cerebrovasc Dis. 2014 Dec 15.

11. Shibazaki K, Kimura K, Okada Y, Iguchi Y, Uemura J, Terasawa Y, Aoki J. Plasma brainnatruiretic peptide as an independent predictor of in-hospital mortality after acute ischemic stroke. Intern Med.2009;48(18):1601-6.
12. Chen $X$, zhan $X$, Chen $M$, Lei $H$, Wang $Y$, Wei D, Jiang $X$. The prognostic value of combined NT-proBNP levels and NIHSS scres in patients with acute ischemic stroke. Intern Med. 2012;51(20):2887-92

13. Montaner J, Garcia-Berrocoso $\mathrm{T}$, Mendioroz M, Palacios M, Perea-Gainza M, Delgado $\mathrm{P}$ et al, Brain natriuretic peptide is associated with worsening and mortality in acute stroke patientsbut adds no prognostic value to clinical predictors of outcome. Cerebrovasc Dis. 2012;34(3): 240-5

14. Nigro N, Wildt $K$, Mueller C, Schuetz $\mathrm{P}$, Mueller B,. Fluri F, et al. BNP but not s-c Tnin is associated with cardioembolie aetiology and predicts short and long term prognosis after cerebrovascular events. PLos One 2014;9(7):e102704

15. Jensen JK, Atar D, Kristensen SR, Mickley H, Januzzi Jl. Usefulness of natriuretic peptide testing for long term risk assessment following acute ischemic stroke. Am J Cardiol 2009 Jul 15; 104 (2):287-91.

16. $\mathrm{Tu} \mathrm{WJ}^{1}$, Dong $\mathrm{X}$, Zhao SJ, Yang DG, Chen H.Prognostive valueof plasma neuroendocrine biomarkers in patients with acute ischemic stroke. J Neuroendocrinol 2013 Sep;25 (9):771-8.

17. Maruyama, Shiga T, Iijima M, Moriya $\mathrm{S}$, Mizuno S, Toi $\mathrm{S}$ et al. Brain natriuretic peptide in acute ischemic stroke. J Stroke Cerebrovasc Dis 2014 May-Jun;23 (5):967-72

18. Sakamoto $Y$, Sato $S$, Kuronuma $Y$, Nagatsuka K, Minematsu K, Toyoda K. Factors associated with proximal carotid axis occlusion in patients with acute stroke and atrial fibrillation. . J Stroke Cerebrovasc Dis 2014 May-Jun;23 (5):799-804

19. Kerola $T$, Nieminem $T$, Hartikainen $S$, Sulkava R, Vuolteenaho O, Kettunen R. Btype natriuretic peptide as a predictor of declining cognitive function and dementia - a cohirt study of an elderly general population with a 5-year follow-up. Ann.Med.2010 Apr;42(3): 207-15. 